\title{
Independent component analysis applied to long bunch beams in the Los Alamos Proton Storage Ring
}

\author{
Jeffrey S. Kolski, ${ }^{*}$ Robert J. Macek, Rodney C. McCrady, and Xiaoying Pang \\ Los Alamos National Laboratory, Los Alamos, New Mexico 87545, USA
}

(Received 29 September 2011; published 27 November 2012)

\begin{abstract}
Independent component analysis (ICA) is a powerful blind source separation (BSS) method. Compared to the typical BSS method, principal component analysis, ICA is more robust to noise, coupling, and nonlinearity. The conventional ICA application to turn-by-turn position data from multiple beam position monitors (BPMs) yields information about cross-BPM correlations. With this scheme, multi-BPM ICA has been used to measure the transverse betatron phase and amplitude functions, dispersion function, linear coupling, sextupole strength, and nonlinear beam dynamics. We apply ICA in a new way to slices along the bunch revealing correlations of particle motion within the beam bunch. We digitize beam signals of the long bunch at the Los Alamos Proton Storage Ring with a single device (BPM or fast current monitor) for an entire injection-extraction cycle. ICA of the digitized beam signals results in source signals, which we identify to describe varying betatron motion along the bunch, locations of transverse resonances along the bunch, measurement noise, characteristic frequencies of the digitizing oscilloscopes, and longitudinal beam structure.
\end{abstract}

DOI: 10.1103/PhysRevSTAB.15.112802

PACS numbers: 29.27. $-\mathrm{a}, 29.85 .-\mathrm{c}$

\section{INTRODUCTION}

Independent component analysis (ICA) is a powerful blind source separation (BSS) method [1]. ICA's major advantage over the typical BSS method, principal component analysis (PCA), which is the BSS foundation of the well-known model independent analysis (MIA) [2], is that it is more robust to noise, coupling, and nonlinearity [3-5]. Because of its robustness and generality, ICA has been widely applied in different fields, such as image feature extraction, audio separation, brain imaging, telecommunications, and econometrics [1], but using ICA for beam analysis is relatively new [3-8].

ICA and PCA separate data into source signals without a beam dynamics model or knowledge of measurement details. In PCA, the separated source signals are uncorrelated, while the source signals from ICA are independent, which mathematically is a much stronger property. It is easy to show that uncorrelatedness is not sufficient to cleanly separate source signals [1], especially in the presence of coupling and nonlinearity [3-5].

We are aware of post-BSS processing techniques that complete separation of mixed sources resulting from PCA such as those described in Refs. [9,10]. However, these techniques are not BSS methods. They require additional information from a model and additional assumptions of the source signals other than the original PCA assumption

\footnotetext{
*jkolski@lanl.gov
}

Published by the American Physical Society under the terms of the Creative Commons Attribution 3.0 License. Further distribution of this work must maintain attribution to the author(s) and the published article's title, journal citation, and DOI. of uncorrelatedness. We choose to use ICA for two reasons: no imposed data model and no additional assumptions of the source signals other than independence.

ICA and PCA model data as a linear combination of independent components (ICs) and principal components (PCs), respectively. Each IC consists of a spatial mode and a temporal mode describing a source signal's strength in space and time. Likewise, each PC possesses a spatial pattern and a temporal pattern that is associated with a source signal's strength in space and time.

Previous application of ICA to beam analysis uses the conventional multibeam position monitor (BPM) scheme, where turn-by-turn position data from multiple BPMs yields information about cross-BPM correlations. MultiBPM ICA has been used to measure the transverse betatron phase and amplitude functions, dispersion function, linear coupling, sextupole strength, and nonlinear beam dynamics [3-5].

We apply ICA in a new way to slices along the single beam bunch in the Los Alamos Proton Storage Ring (PSR, see Table I for relevant parameters). We digitize beam signals of the long PSR bunch with a single device (BPM or fast current monitor) for an entire injection-extraction cycle, nominally 1745 turns. Sufficiently digitized signal bins are spatially distributed along the beam bunch, giving adequate spatial structure and providing ICA with enough raw data. ICA of the digitized beam signals yields correlations of particle motion within a beam bunch and provides a new perspective to investigate beam dynamics within the bunch.

In this paper, we adopt the ICA algorithm second order blind identification (SOBI) [11] that utilizes the time structure of measured beam signals and deciphers the source 
TABLE I. PSR relevant parameters.

\begin{tabular}{ll}
\hline \hline Typical accumulation & 1745 turns $(625 \mu \mathrm{s})$ \\
Extended accumulation & 3420 turns $(1250 \mu \mathrm{s})$ \\
Optional storage & 558 turns $(200 \mu \mathrm{s})$ \\
Betatron tune $\left(\nu_{x}, \nu_{y}\right)$ & $(3.19,2.19)$ \\
LANSCE linac frequency & $201.25 \mathrm{MHz}$ \\
Subharmonic & 72.07 \\
(linac frequency/revolution frequency) & \\
Revolution frequency & $2.792 \mathrm{MHz}$ \\
Revolution period & $358 \mathrm{~ns}$ \\
\hline \hline
\end{tabular}

signals by simultaneous diagonalizing multiple autocovariance matrices, see Appendix C.

We organize this paper as follows: The formalism of ICA and PCA and the nomenclature used in this paper are introduced in Appendices A, B, and C. We describe slices along the bunch and our data collection in Sec. II. In Sec. III, we discuss results of ICA applied to slices along the bunch, including identification and experimental verification of several source signals. The conclusions regarding ICA of slices along the bunch are given in Sec. IV.

\section{ICA OF SLICES ALONG LONG BUNCH BEAMS}

In previous applications to beam analysis, ICA is applied to data from multiple BPMs [3-6]. We apply ICA in a new way to slices along the bunch [6-8]. We digitize beam signals from a single device (BPM or fast current monitor) for a full injection-extraction cycle. We divide the digitized signal into slices of equal length using the $0.5 \mathrm{~ns}$ digitization bin length. The long digitized signal vector is stacked turn by turn to form the data matrix $\mathbf{x}$,

$$
\mathbf{x}(t)=\left(\begin{array}{cccc}
x_{1}(1) & x_{1}(2) & \ldots & x_{1}(N) \\
x_{2}(1) & x_{2}(2) & \ldots & x_{2}(N) \\
\vdots & \vdots & \ddots & \vdots \\
x_{M}(1) & x_{M}(2) & \ldots & x_{M}(N)
\end{array}\right),
$$

such that each row of $\mathbf{x}$ is beam signal from a single slice and each column of $\mathbf{x}$ is beam signal from a single turn. In this picture, turn number is the temporal variation, and slice number is the spatial variation. The last turn beam profile plotted in Fig. 1 is an example of the last column of $\mathbf{x}$.

The slices are located at a fixed longitudinal phase or position along the bunch. The spatial mode describes the IC's strength along the bunch. The ICs produced by ICA applied to slices along the bunch describe longitudinal beam structure and motion that varies along the bunch.

In time series ICA, source signal independence translates to source signals with nonoverlapping power spectra [11]. Source signals with overlapping power spectra lead to redundant singular values (SVs). The SVs from the SOBI algorithm whitening, Eq. (C5), form a continuum of SVs with small but significant magnitude, Fig. 2. There is no

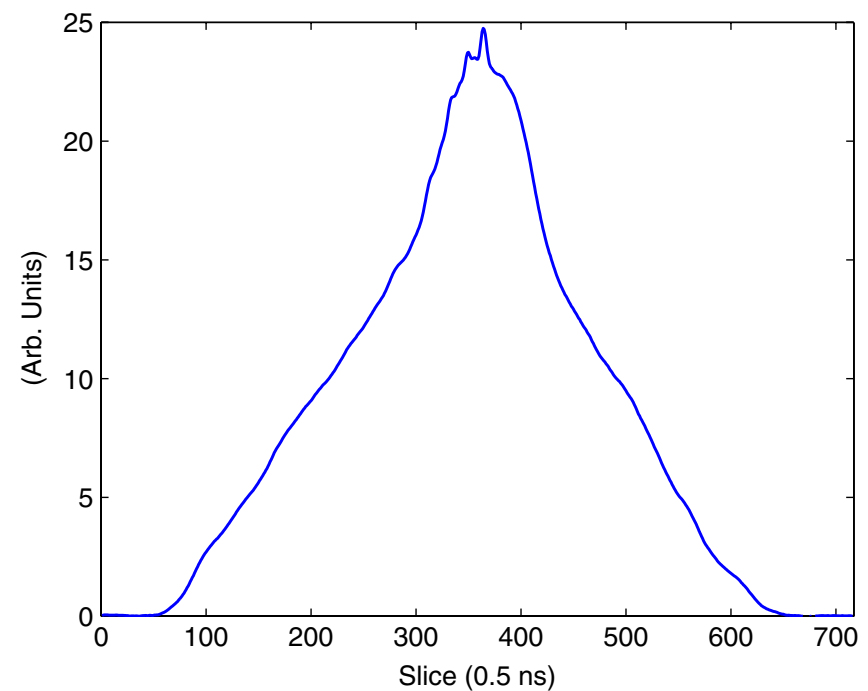

FIG. 1. The last turn beam profile as an example of the last column of the data matrix $\mathbf{x}$ in Eq. (1). The PSR's revolution period is $358 \mathrm{~ns}$ corresponding to 716 slices.

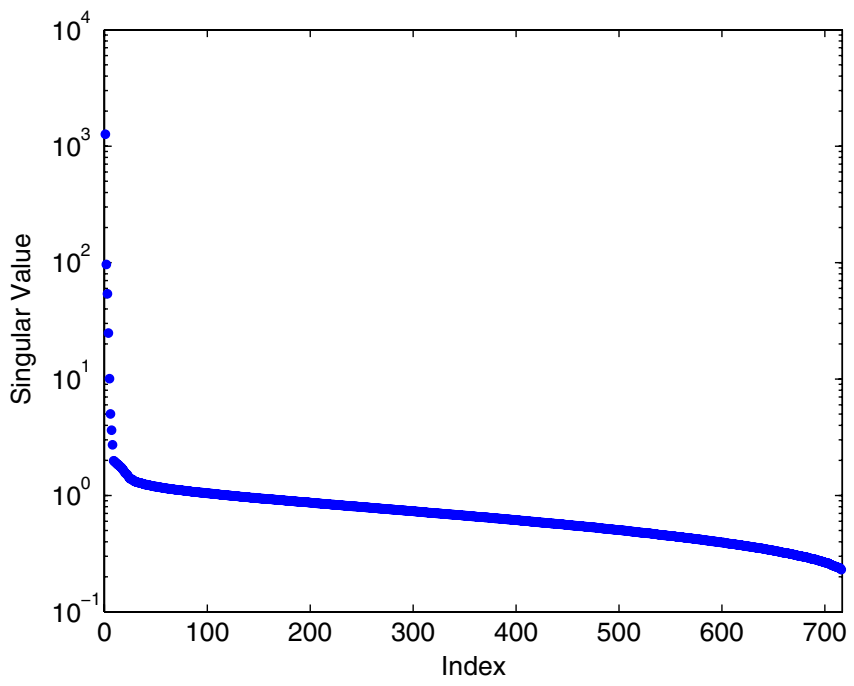

FIG. 2. A typical SV distribution when applying ICA to slices along the bunch. The SVs are calculated during the whitening portion of the SOBI algorithm, Eq. (C5).

obvious cutoff threshold $\lambda_{c}$ to limit the redundancy of the data, and some source signals possess SVs in the continuum, so we must include many SVs from the continuum in our analysis. This is quite different than applications of multi-BPM ICA and multi-BPM PCA, where only a few SVs are significant [2-6].

We digitize beam signals from a fast current monitor, SRWC41 [12], and a short stripline BPM with $400 \mathrm{MHz}$ peak frequency response, SRWM41. We digitized SRWM41's horizontal and vertical sum and difference signals for ICA. The integral of the sum and difference signals are calculated offline and also analyzed by ICA.

ICA can be applied to individual beam signals or combinations of signals to investigate coupling between 
horizontal and vertical planes. In this paper, we only report results of ICA applied to a single beam signal. We typically ran our analysis for $L=30 \mathrm{SVs}, K=50$ time lags, and as many turns as possible.

\section{INDEPENDENT COMPONENTS}

We apply ICA to the digitized beam signals from SRWC41 and SRWM41. The ICs resulting from ICA need to be related to a physical source. Since the longitudinal dynamics in the PSR are very complex and ICA is a numerical procedure, there is no analytical model to assign ICs to a physical source. We resort to measurements to gain understanding of the ICs. In this section, we identify and experimentally verify several ICs from ICA applied to slices along the bunch.

The ICs presented in this section are represented by a series of graphs; see Fig. 3 as an example. The top left graph plots the spatial mode (blue) with the last turn beam profile (green).

The bottom left graph plots the fast-Fourier-transform (FFT) of the spatial mode (top left) and reports the peak integer revolution harmonic. The spatial mode FFT is over one revolution, so the resolution is limited to an integer $(1 / 1)$.

The top center graph plots the integrated spatial mode (top left) (blue) and the last turn beam profile (green). Integrating the spatial mode can lead to better IC interpretation. We plot the integrated spatial mode only for ICs derived from SRWM41's sum and difference signals.

The bottom center graph plots the correlation of the IC's mean-zero signal constructed via Eq. (C11) and each timelagged, whitened data matrix $\mathbf{z}(t-\tau)$. The SV from Eq. (C5) is reported.

The top right graph plots the temporal mode, where turn -1 is the last turn. The fractional revolution harmonic result from a sinusoid fit is quoted.
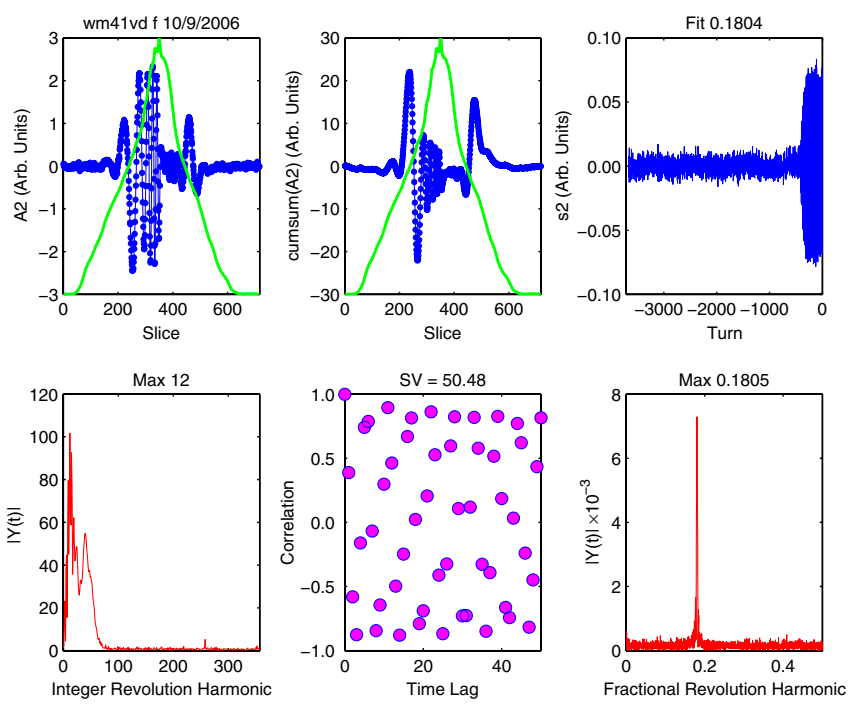

FIG. 3. A typical betatron IC resulting from ICA of SRWM41's vertical difference signal for a single-turn kick.
The bottom right graph plots the FFT of the temporal mode (top right) and indicates the peak fractional revolution harmonic. The temporal mode FFT resolution is equal to one over the number of turns $(1 / N)$.

The IC's total revolution harmonic is the sum of the integer and fractional revolution harmonics. The frequency of the IC's signal is the product of total revolution harmonic and revolution frequency.

\section{A. Betatron ICs}

ICA's ability to obtain quality ICs representing betatron motion is well established in previous applications of multiBPM ICA [3-5]. It is of interest to examine ICA's betatron motion result when applied to slices along the bunch. The beam must undergo coherent betatron oscillation, which we induce with a vertical single-turn kick $50 \mu$ s into a store time after extended accumulation. The beam is stored for 420 turns $(150 \mu \mathrm{s})$ after the single-turn kick.

The IC in Fig. 3 is derived from SRWM41's vertical difference signal and is identified as betatron motion because the fractional revolution harmonic (bottom right) is close to the operating fractional betatron tune value and because the temporal mode (top right) is only nonzero for turns after the single-turn kick. For betatron motion ICs, the fractional revolution harmonic equals the fractional betatron tune, but the integer revolution harmonic is not equivalent to the integer betatron tune.

The spatial mode (top left) has units proportional to the derivative of current and indicates the strength of the 0.1805 fractional betatron tune oscillation along the beam bunch. In this case it is easier to interpret the integrated spatial mode (top center) because it has units proportional to current. The integrated spatial mode maximums indicate that the majority of particles undergoing coherent betatron motion with a fractional tune of 0.1805 are located symmetric about the bunch center at slices 237 and 474 and represent the coherent space charge tune shifted beam. The fast oscillation slightly forward of the bunch center describes mixing of betatron tunes for the central time slices.

In theory, each slice possesses a slightly different frequency due to the coherent space charge tune shift. If the analysis includes ample time lags, ICA could recover a distinct IC for each slice. From our analysis using $30 \mathrm{SVs}$ and 50 time lags, we identify 15 betatron ICs.

We wish to compare the betatron ICs with the data matrix FFT. We define the greatest strength location to be the fractional revolution harmonic and slice where an IC is strongest. The slice coordinate is determined by the integrated spatial mode (top center) maximum. We calculate a leading and trailing edge greatest strength location for each IC.

In Fig. 4, we compare the greatest strength locations of the first 30 ICs with the tune distribution given by the data matrix FFT along turn for each slice. The greatest strength locations of the 15 betatron ICs reproduce the tune distribution. The spatial mode (top left) in Fig. 3 replicates the 


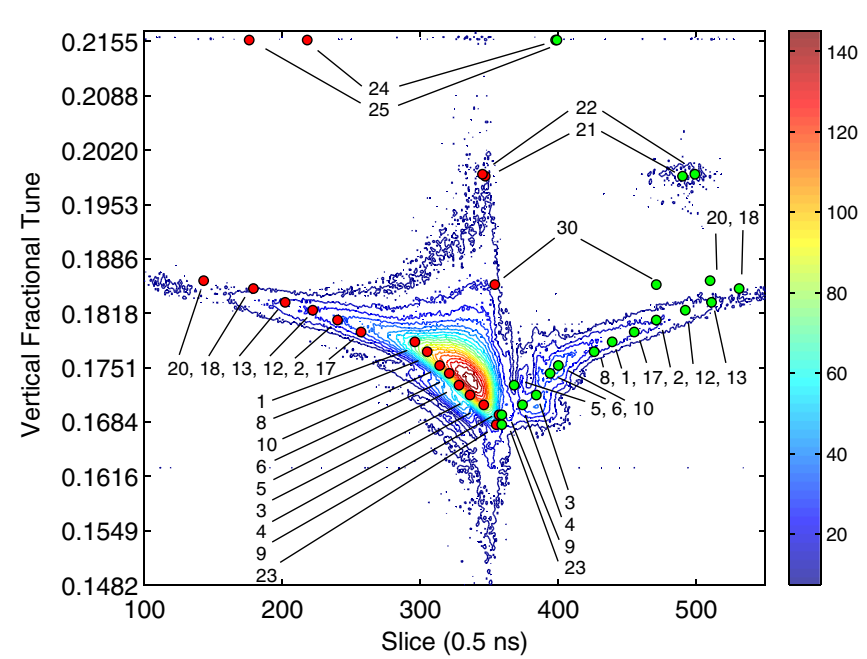

FIG. 4. Betatron tune along the bunch and the greatest strength location of the first $30 \mathrm{ICs}$, some of which lie outside the plot boundary. The contour plots the data matrix FFT along the turn for each slice. The red and green circles mark the leading and trailing edge greatest strength locations, respectively, with IC number indicated by numeric labels. The beam profile peak is located at slice 350 .

0.1805 fractional tune contour along the bunch of the tune distribution in Fig. 4. There is no one IC that describes the entirety of the coherent space charge tune shift. Although the betatron ICs do not represent independent motion, they do describe real beam motion. The betatron ICs must be viewed in concert as in Fig. 4 to obtain the full picture of the coherent space charge tune shift along the bunch.

Previous applications of multi-BPM ICA result in a single tune measurement. ICA applied to slices along the bunch yields a fuller picture of the particle dynamics for long bunch beams.

Now for comparison, we apply PCA to the same data matrix. A betatron PC is plotted in Fig. 5, which plots similar quantities as the IC graphs. The IC (Fig. 3) and PC are both component number 2 and possess the same SV, but this does not imply that they describe the same source signal. The PC in Fig. 5 describes a 0.1751 fractional tune oscillation along the bunch.

The integrated spatial pattern indicates that the greatest strength location of the 0.1751 fractional tune oscillation is at the bunch center. The strength of the PC decays symmetrically about the peak current.

The full width half maximum of the temporal pattern FFT (bottom right) peak is twice the ICA result in Fig. 3 (0.0063 compared to 0.0032) indicating that PCA is less able to separate nearby frequencies.

The most significant difference between the ICA and PCA result is that all of the betatron PCs are mixed with other source signals. An example of coupling can be seen in the temporal pattern FFT (bottom right) in Fig. 5, which shows two distinct peaks at fractional revolution harmonics 0.1751 and 0.38 . The source signal mixing is small in Fig. 5
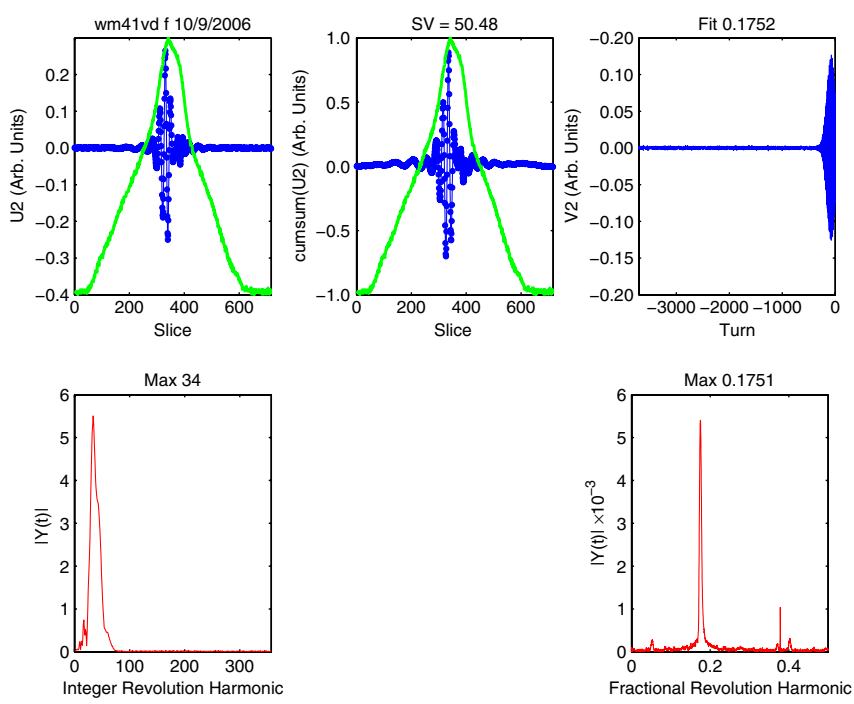

FIG. 5. A typical betatron PC resulting from PCA of SRWM41's vertical difference signal for a single-turn kick.

as indicated by the temporal pattern FFT and has little effect of the spatial pattern. Sometimes the coupling is not small as for the PC plotted in Fig. 6.

The PC shown in Fig. 6 has dominant fractional revolution harmonic (bottom right) 0.46 . The broad betatron peak in the temporal pattern FFT (fractional revolution harmonics 0.16-0.20) indicates that this PC mixes with much of the tune continuum. The spatial pattern (top left) is a mixture of betatron motion and the dominant source signal, which we will describe latter in Sec. III D 1. The substance of this PC is corrupted by the incomplete source separation of PCA.

We compare the PCA result with the data matrix FFT in Fig. 7, which is similar to Fig. 4 except plots the greatest strength locations for the first 30 PCs. We include in Fig. 7
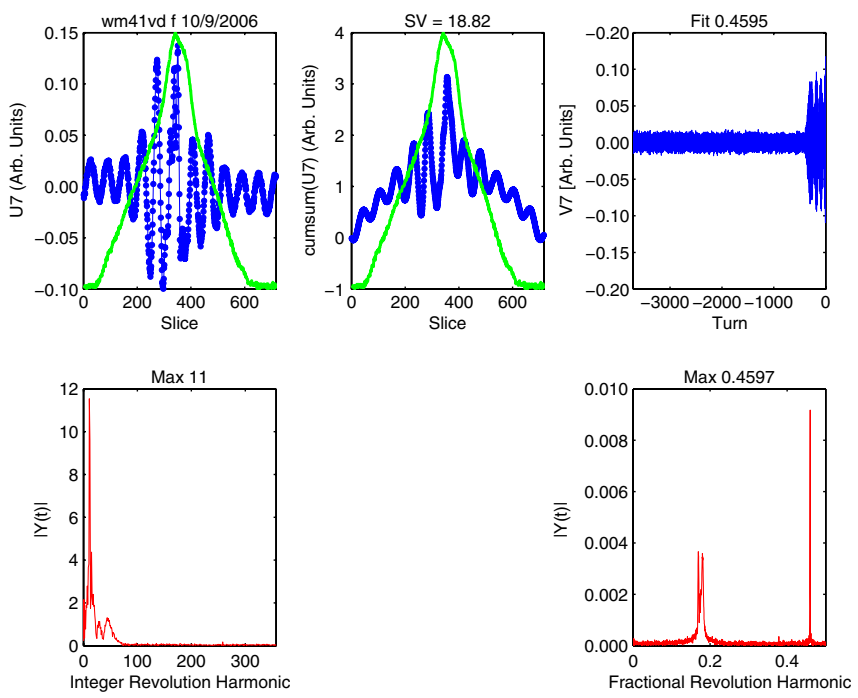

FIG. 6. Another betatron PC resulting from PCA of SRWM41's vertical difference signal for a single-turn kick. 


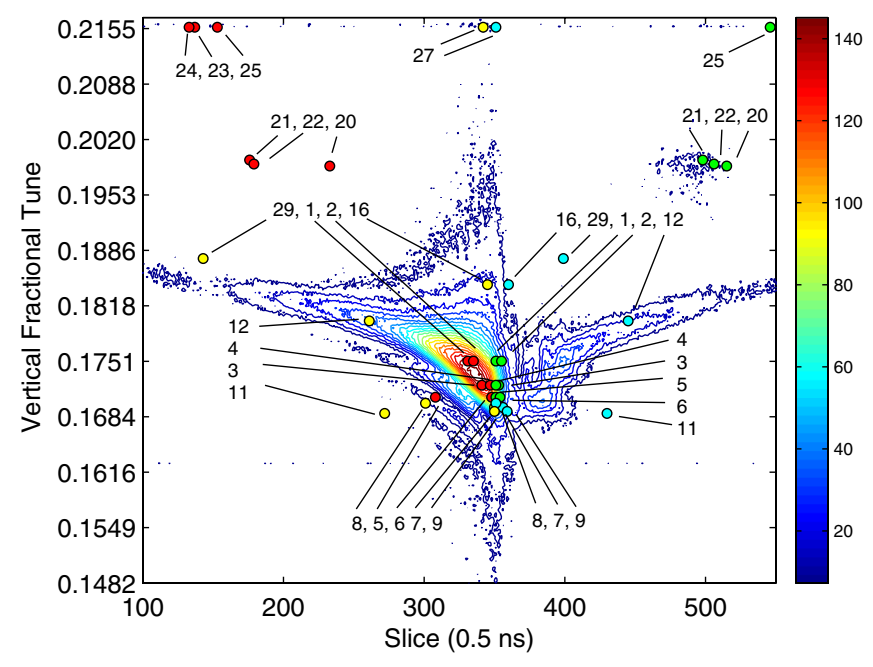

FIG. 7. Betatron tune along the bunch and the greatest strength location of the first $30 \mathrm{PCs}$, some of which lie outside the plot boundary. The contour plots the data matrix FFT along the turn for each slice. The red and green circles mark the leading and trailing edge greatest strength locations, respectively, for PCs with dominant betatron motion. The yellow and cyan circles show the leading and trailing edge greatest strength locations, respectively, for PCs with a dominant source signal other than betatron motion. A numeric label indicates the PC number. The beam profile peak is located at slice 350 .

both PCs dominated by betatron motion like in Fig. 5 and PCs dominated by a source signal and coupled with betatron motion like in Fig. 6. All betatron PCs have peak strengths located near the bunch center where the vertical difference signal is largest because PCA is unable to diagonalize the frequency continuum beyond its peak strength and average location. It is clear from Fig. 7 that PCA is unable to recover the coherent space charge tune shift along the bunch.

\section{B. Resonance ICs}

We also identified resonance ICs from the single-turn kick experiment. The resonance ICs are labeled component number 21 and 22 in Fig. 4, where the fifth order resonance island is located between slices 470 and 510. IC number 21 is plotted in Fig. 8.

We identify the IC as a fifth order resonance because the temporal mode (top right) grows exponentially and because of the sharp 0.2 temporal mode FFT peak (lower right). An oscillation with fractional tune of 0.2 has period of 5 turns, which is observed in the correlation between the resonance IC's constructed signal and the time-lagged, whitened data (bottom center). The correlation maximizes every five time lags (each time lag is one turn) as the whitened time-lagged data shifts in and out of phase with the resonance IC's constructed signal. The decay in the correlation with time lag is due to weaker resonance strength for earlier turns.
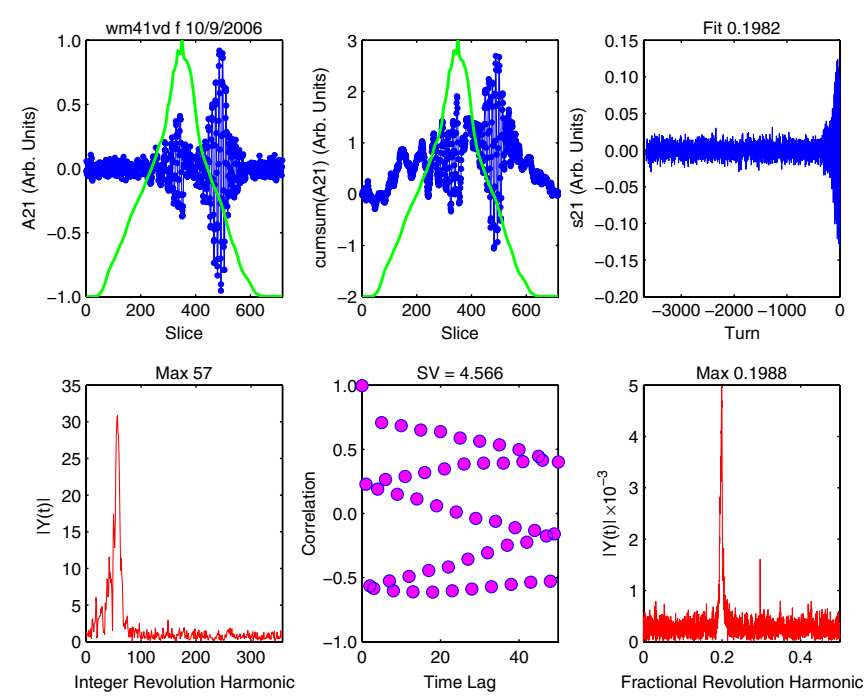

FIG. 8. A typical resonance IC resulting from ICA of SRWM41's vertical difference signal for a single-turn kick.

The spatial mode (top left), which is a good representation of the 0.2 fractional tune contour from the tune distribution in Fig. 4, locates the majority of particles undergoing resonance on the trailing edge for slices 470-510, coincident with the resonance island.

The exponential growth time constant $T$ for the resonance IC can be estimated by fitting an exponential function $\left(e^{n / T}\right.$, where $n$ is a turn index) to the temporal mode envelope. Applying the exponential fit to turns after the single-turn kick yields $T=150$ turns or $54 \mu \mathrm{s}$. $T$ is the time constant for the entire motion described by the resonance IC, which contains frequencies of the 0.2 sidebands of revolution harmonics 10-65 in proportions represented by the spatial mode FFT (lower left). The time constant calculated by ICA is a more complete picture than that of a single frequency calculated by FFT.

\section{Noise ICs}

Not all ICs represent beam motion, some describe measurement noise. An example noise IC from SRWM41's integral vertical sum signal is plotted in Fig. 9. We identify the IC as noise because the correlation of the IC's constructed signal and the time-lagged, whitened data (bottom center) only possesses significant correlation with the zero time-lagged data and because the temporal mode FFT (bottom right) results in a frequency spectrum with no distinct frequency peaks. Noise ICs also possess a large spatial mode (top left) for time slices where beam is not present and a constant amplitude temporal mode (top right), confirming that the IC does not represent beam motion.

Using a PCA result, we can estimate the rms standard deviation of the noise source from the SV. The SV from PCA of random, mean-zero, Gaussian noise is

$$
\Lambda_{i}=\sigma_{i} \sqrt{N}
$$



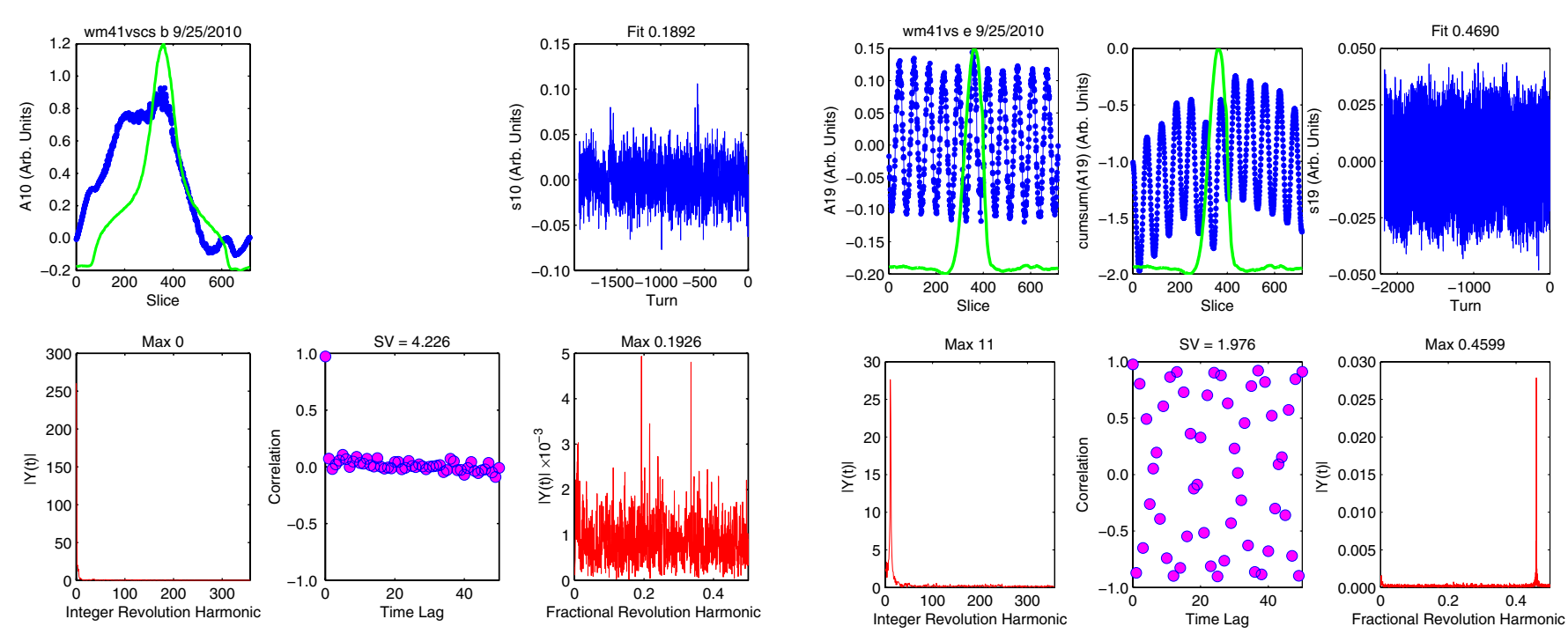

FIG. 9. A typical noise IC resulting from ICA of SRWM41's integral vertical sum signal.

where $\Lambda$ is the SV, $\sigma$ is the noise source's rms standard deviation, $N$ is the number of turns, and $i$ is the index of the $i$ th noise source. A SV of 4.226 (bottom center) yields $\sigma=$ 0.0949 arbitrary units. $\sigma$ has the same units as all spatial modes derived from the same beam signal.

\section{Oscilloscope ICs}

Sources of oscilloscope ICs originate from the digitizing oscilloscope. Oscilloscope ICs contain constant amplitude sinusoidal spatial and temporal modes, which indicate that they do not represent beam motion. They also possess oscillatory correlation with the time-lagged, whitened data. Oscilloscope ICs possess unusually sharp peaks in both their spatial and temporal mode FFTs indicating that they describe constant frequency components of the digitized signal.

Oscilloscope ICs are always produced in pairs. ICA regards a phase shifted sinusoidal signal as two sources as in the sine angle addition formula,

$$
\begin{aligned}
& \sin [2 \pi f n(t)+\phi(x)] \\
& \quad=\cos [\phi(x)] \sin [2 \pi f n(t)]+\sin [\phi(x)] \cos [2 \pi f n(t)],
\end{aligned}
$$

where $f$ is a frequency, the turn index $n$ is a function of time $t$ (temporal mode), and the phase $\phi$ is a function of space $x$ (spatial mode). The phase shift describes a slippage in the source signal's initial phase each turn since the beam signal is stacked according to the revolution frequency, which in general is not an integer subharmonic of the source signal. The pair ICs possess identical spatial and temporal mode FFTs. The pair ICs' spatial and temporal modes only differ by a phase associated with a cosine and sine-type oscillation.

Since their strength is an order of magnitude or more weaker compared to the strongest source signal, the

FIG. 10. A typical oscilloscope clock IC resulting from ICA of SRWM41's vertical sum signal.

interesting aspect about the oscilloscope IC result is ICA's capability to separate weak independent source signals.

\section{Oscilloscope clock ICs}

We identify the IC in Fig. 10 as an oscilloscope clock IC for reasons that will become clear later. The oscilloscope clock IC is only produced from beam signals digitized by our Lecroy LC684DXL oscilloscope. When the same beam signals are digitized with our Lecroy LC584AXL oscilloscope, the oscilloscope clock IC is not observed. This suggests that the oscilloscope clock IC represents something internal to the Lecroy LC684DXL oscilloscope.

Concluding the oscilloscope clock IC describes internal workings of the oscilloscope helps to explain the precise nature of the sinusoidal spatial mode (top left). The spatial mode performs just under 11.5 oscillations in a turn. The number of oscillations per turn may also be calculated by adding the integer (bottom left) and fractional revolution harmonics (bottom right).

We need to consider the ambiguity of the fractional revolution harmonic with respect to the half integer. In this example the fractional revolution harmonic can be verified visually from the spatial mode (top left), but in general this ambiguity needs resolution before the total revolution harmonic is obtained.

The best and most reliable manner of determining an IC's total revolution harmonic is to use the IC's constructed signal. However, if the IC is identified as a pair, constructing the signal via Eq. (C11) will only yield half of the source signal. We must combine Eqs. (C11) and (3) to construct the signal described by the pair ICs,

$$
\mathbf{z}_{\mathrm{IC}_{i, j}}=\vec{A}_{i} \cdot \vec{s}_{i}+\vec{A}_{j} \cdot \vec{s}_{j},
$$


where $i$ and $j$ are pair ICs. Once $\mathbf{z}_{\mathrm{IC}_{i, j}}$ is constructed, it must be unstacked. An FFT of the ICs' constructed signal vector will yield the total revolution harmonic for both ICs.

An FFT of the oscilloscope clock ICs' constructed signal vector yields a total revolution harmonic of 11.4599. The frequency of the oscilloscope clock IC, calculated by multiplying the revolution frequency by 11.4599 , is an exact $32 \mathrm{MHz}$, indicating precision expected of a digital electronic system. The $32 \mathrm{MHz}$ source internal to the LeCroy LC684DXL oscilloscope is most likely a $32 \mathrm{MHz}$ bus clock the processor uses to make $96 \mathrm{MHz}$ [13].

Direct evidence of the $32 \mathrm{MHz}$ oscilloscope clock IC is found in the FFT of the digitized beam signal, Fig. 11, which shows the revolution harmonics separated by the revolution frequency. The pattern is broken by the peak at $32 \mathrm{MHz}$. The FFT confirms that the $32 \mathrm{MHz}$ signal is an independent source signal, possessing a nonoverlapping power spectrum. The $32 \mathrm{MHz}$ signal is not a revolution harmonic or harmonic of another IC.

The $32 \mathrm{MHz}$ signal has been observed for several years in FFTs of SRWM41's sum and difference signals, which are traditionally digitized with the LeCroy LC684DXL oscilloscope, but its source was not identified because the FFT signal analysis approach only provides the source signal frequency. With the additional spatial and temporal mode information provided by ICA, we identify the source of the oscilloscope clock IC with a $32 \mathrm{MHz}$ clock internal to the LeCroy LC684DXL oscilloscope.

\section{Oscilloscope digitizer ICs}

The oscilloscope digitizer IC is also associated with the digitizing oscilloscope. An oscilloscope digitizer IC produced from ICA of SRWC41's fast current signal is plotted in Fig. 12. The oscilloscope digitizer IC shows some dependence on the beam intensity and results from ICA of every beam signal.

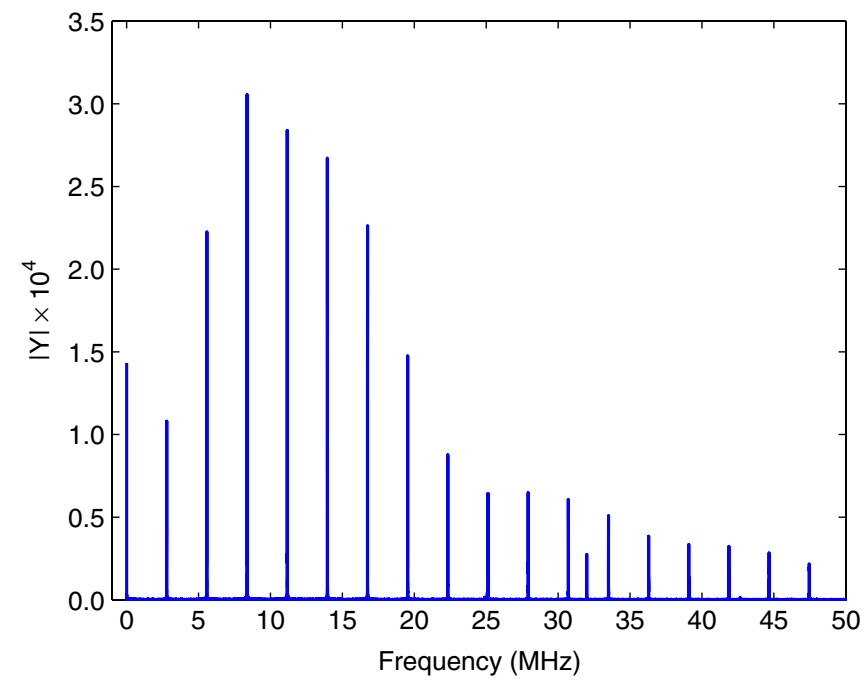

FIG. 11. Frequency spectrum of the SRWM41 vertical sum signal that produced the oscilloscope clock IC in Fig. 10.
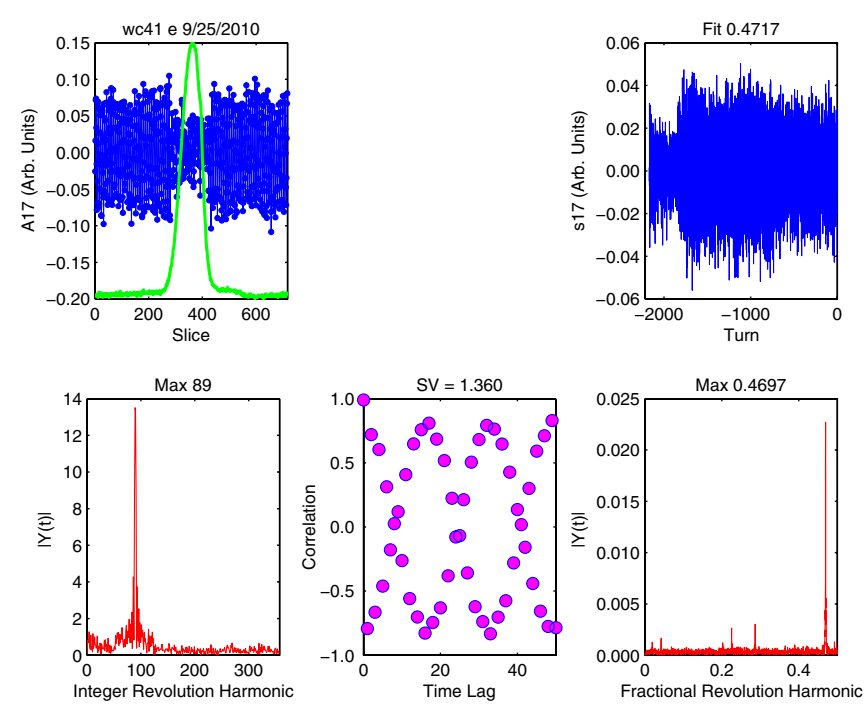

FIG. 12. A typical oscilloscope digitizer IC resulting from ICA of SRWC41's fast current signal.

We use the procedure described in Sec. IIID 1 to resolve the fractional revolution harmonic's ambiguity about the half integer. The reconstructed data vector FFT yields a total revolution harmonic of 89.5303. The frequency of the oscilloscope digitizer IC, calculated by multiplying the revolution frequency by 89.5303 , is an exact $250 \mathrm{MHz}$.

The beam signals are digitized at $2 \mathrm{GS} / \mathrm{s}$ on LeCroy LC584AXL and LC684DXL oscilloscopes, which interleave four $500 \mathrm{MHz}$ digitizers to obtain the sampling rate. The individual digitizer sample rate is $500 \mathrm{MS} / \mathrm{s}$, and the digitizer Nyquist frequency is $250 \mathrm{MHz}$. The oscilloscope digitizer IC is an artifact of four $500 \mathrm{MHz}$ digitizers interleaving to obtain a $2 \mathrm{GS} / \mathrm{s}$ sample rate [13].

\section{E. 201.25 MHz ICs}

The 201.25 MHz IC is the first IC we identified. It represents the $201.25 \mathrm{MHz}$ longitudinal beam structure, established by the LANSCE linac during acceleration, of beam newly injected in the PSR. The 201.25 MHz IC is produced by every beam signal but is best observed in SRWM41's vertical sum signal, Fig. 13.

The $201.25 \mathrm{MHz}$ IC is identified by the character of its temporal mode (top right), which has constant amplitude for the first 1400 turns and suddenly reduces to noise for the last 500 turns. The reduction coincides with the end of accumulation and the beginning of storage, indicating that the $201.25 \mathrm{MHz}$ IC describes newly injected beam.

The $201.25 \mathrm{MHz}$ ICs are produced in pairs, so we verify the IC's frequency using the procedure in Sec. III D 1. The total revolution harmonic is 72.07136 as expected because the PSR design revolution frequency is the 72.07 subharmonic of the $201.25 \mathrm{MHz}$ linac frequency. Multiplying the total revolution harmonic by the revolution frequency yields exactly $201.25 \mathrm{MHz}$. 

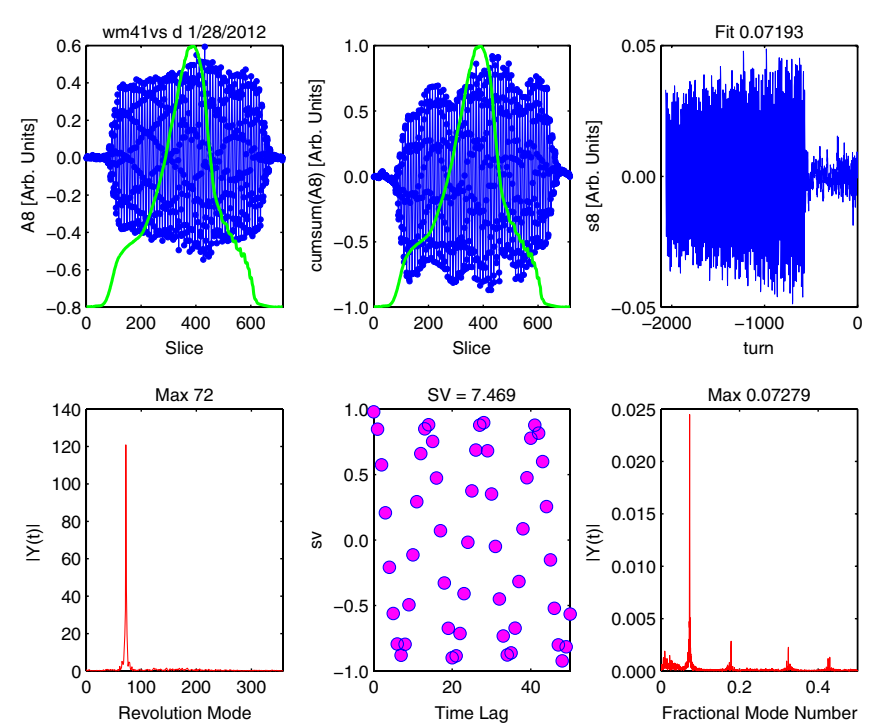

FIG. 13. A typical 201.25 MHz IC resulting from ICA of SRWM41's vertical sum signal.

The power of ICA, which lies in the spatial mode (top left), is most prominent for the $201.25 \mathrm{MHz}$ IC. The spatial mode is constant amplitude across the injection region and zero outside. The spatial mode describes the injection length each turn (pattern width). As we will show, the spatial mode also describes the longitudinal phase of the injected beam.

The spatial mode is a sinusoidal oscillation with period $5 \mathrm{~ns}$, a $201.25 \mathrm{MHz}$ period, and 58 peaks. Each peak represents a single linac pulse (linac rf bucket) injected into the PSR. The injection pattern width is $290 \mathrm{~ns}$, which divided by the $201.25 \mathrm{MHz}$ period yields 58 linac pulses injected per turn as indicated by the spatial mode peaks.

The spatial mode's (top left) constant amplitude indicates that the charge in each linac pulse does not vary significantly across a turn. One might expect the spatial mode to be positive like the newly injected beam current, but the SOBI algorithm's preprocessing constrains the spatial mode to have an average of zero.

We now examine additional examples of the spatial mode (top left) produced from beam signals collected under different injection schemes. The 201.25 MHz IC plotted in Fig. 14 was produced from beam signal collected while injecting a $50 \mathrm{~ns}$ pattern width beam $120^{\circ}$ early in the PSR's rf bucket. The spatial mode shows an injection region 100 slices wide, corroborating the pattern width. Additionally, the spatial mode's position has shifted forward, confirming injection early in phase. The last turn profile indicates that after $200 \mu \mathrm{s}$ of storage the majority of beam is located opposite in the rf bucket of where it was injected.

The 201.25 MHz IC plotted in Fig. 15 was produced from beam signals collected while injecting with a "notch" in the beam, illustrated by the spatial mode (top left). In this example, we used the following notch
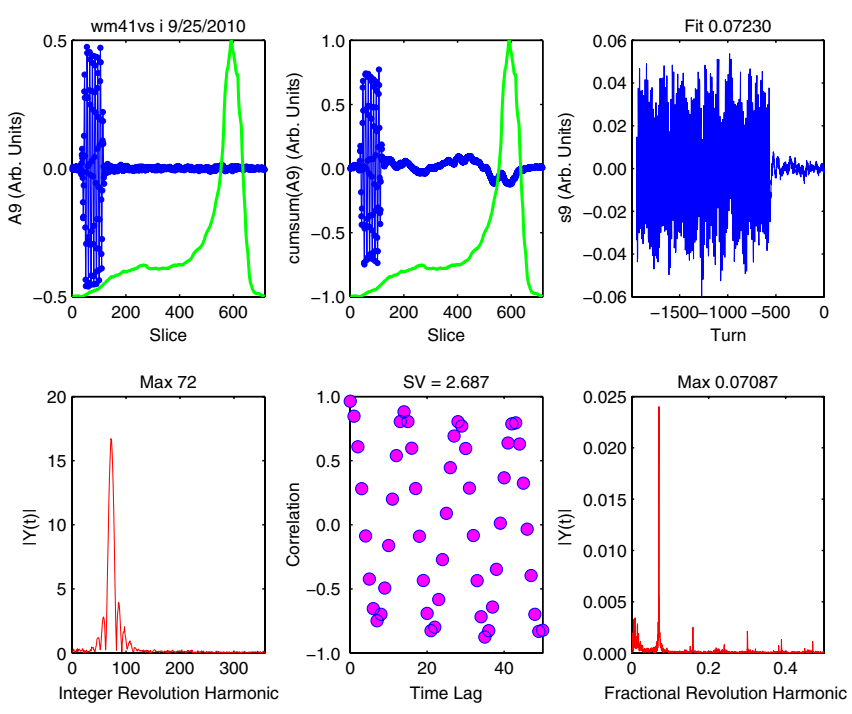

FIG. 14. The 201.25 MHz IC resulting from ICA of SRWM41's vertical sum signal when injecting a $50 \mathrm{~ns}$ pattern width beam $120^{\circ}$ early in the PSR's rf bucket.
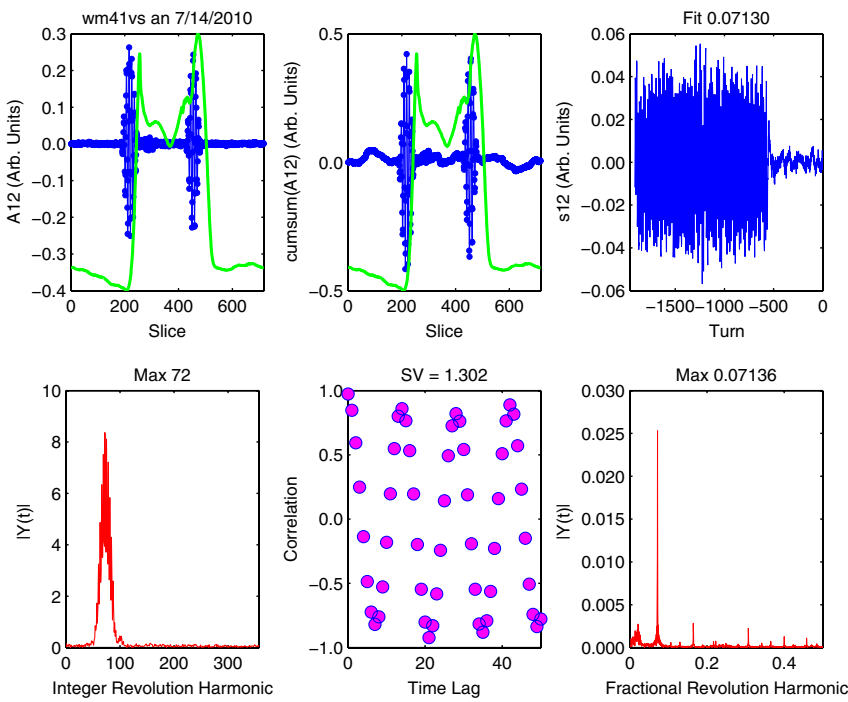

FIG. 15. The 201.25 $\mathrm{MHz}$ IC resulting from ICA of SRWM41's vertical sum signal when injecting beam with a notch.

injection scheme: inject for $30 \mathrm{~ns}$, no injection for $90 \mathrm{~ns}$ (notch), and inject for $30 \mathrm{~ns}$. The spatial mode describes an injection region as two widths of 60 slices separated by 180 slices, thus replicating the notch injection scheme.

The following is an example of the power of ICA and the 201.25 MHz IC. At the beginning of the 2010 LANSCE production run cycle, we observed an unusual amount of "hash" noiselike structure on the PSR beam profile, Fig. 16. The hashy beam profile is an operational concern because it could excite longitudinal microwave instabilities and other longitudinal space charge effects $[14,15]$. 


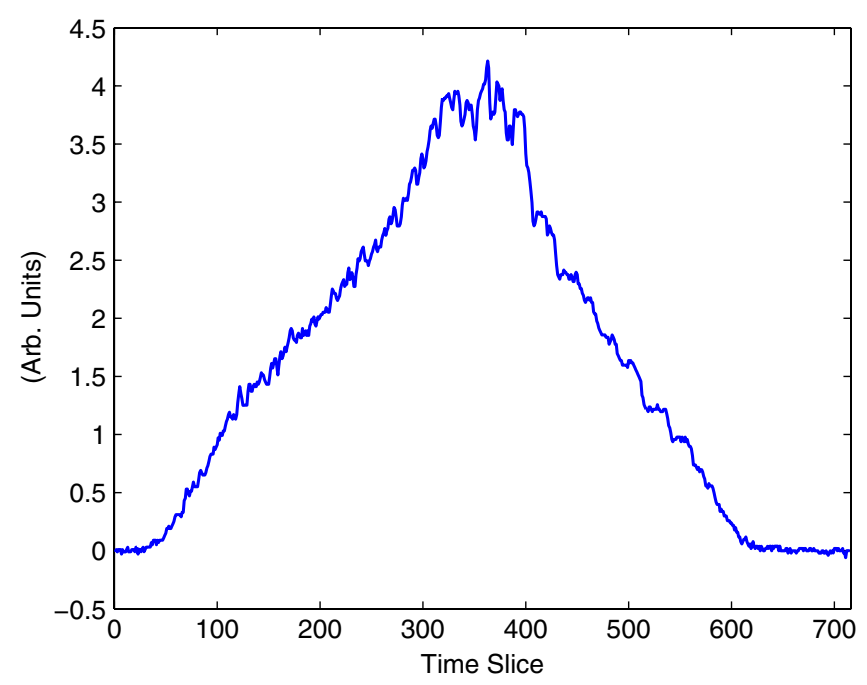

FIG. 16. The last turn current profile of the PSR beam when the revolution frequency is near an integer subharmonic of the linac frequency.

ICA of the hashy beam signal yields the $201.25 \mathrm{MHz}$ IC plotted in Fig. 17, which is reminiscent of Fig. 13. The obvious difference between the two ICs lies in the temporal mode (top right). The IC plotted in Fig. 17 possesses such a small fractional revolution harmonic (bottom right) that the temporal mode's sinusoidal behavior is observable. A fractional revolution harmonic of 0.009 (bottom right) describes an oscillation that repeats every 111 turns. The slow injection rastering causes the hashy beam profile by longitudinally stacking the beam upon itself more than in nominal operations where the fractional revolution harmonic is 0.07 , corresponding to a rastering period of 14 turns.
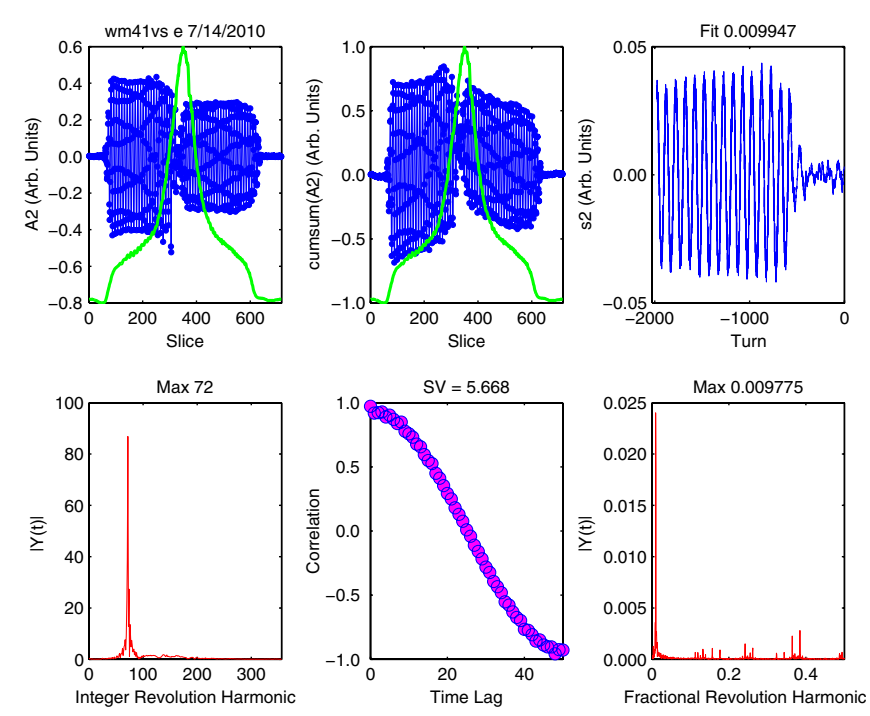

FIG. 17. The 201.25 $\mathrm{MHz}$ IC resulting from ICA of SRWM41's vertical sum signal when the revolution frequency is near an integer subharmonic of the linac frequency.
The spatial mode (top left) in Fig. 17 has a depression in the middle and different constant amplitudes on either side. The depression is not due to an injection notch, but is most likely caused by a quick decoherence of the accumulated beam's $201.25 \mathrm{MHz}$ frequency structure. The spatial mode amplitude difference on either side of center may indicate whether the synchrotron motion of newly injected beam is aligned or antialigned with the rastering injection pattern.

We calculate the PSR revolution frequency from the IC plotted in Fig. 17. The unchanging 201.25 MHz linac frequency is divided by the total revolution harmonic 72.009. This results in a revolution frequency of $2.7948 \mathrm{MHz}$. However, the PSR design revolution frequency is $2.7924 \mathrm{MHz}$, Table I. The revolution frequency differs from design by $2.4 \mathrm{kHz}$ causing the hash on the current profile.

The PSR revolution frequency is created by a frequency generator. A number is typed into a computer to control the revolution frequency. The number inputted into the frequency generator computer was $2.7948 \mathrm{MHz}$, exactly the number predicted by ICA.

\section{CONCLUSIONS}

A new method applying ICA to slices along a long bunch beam is adopted and tested with PSR beam signals. We apply ICA to beam signals of a long bunch with a single device (BPM or fast current monitor) for an entire injection-extraction cycle. In this new method, ICA produces spatial modes describing the ICs' strength along the beam bunch and temporal modes describing the ICs' strength in time (turn). The spatial and temporal modes associated with each IC provide more information about the source signal than an FFT analysis.

We determine that PCA is inadequate for the BSS problem of slices along the bunch because PCA is unable to completely separate the source signals, yielding PCs describing a mixture of two or more source signals.

Six categories of ICs are identified by their spatial and temporal modes and experimentally verified. These include ICs describing the coherent space charge tune shift, a fifth order resonance, measurement noise, a frequency embedded in the digitized beam signal by the digitizing oscilloscope's clock, a signal corresponding to the Nyquist frequency of the digitizing oscilloscope's individual digitizers, and the longitudinal structure of newly injected beam.

We find ICA to be particularly adept at recovering independent source signals from massive amounts of data, even if the sources are weak and in the noise.

Lastly, because of its BSS nature, ICA is able to pick out independent sources without use of a model and provides a great tool for mitigating symptoms of unknown causes. We give an example where ICA was used at PSR to diagnose an off-design revolution frequency, which caused the observed hashy beam profile. 


\section{ACKNOWLEDGMENTS}

We give special thanks to S. Y. Lee of Indiana University for suggesting this problem. This work was supported in part by United States Department of Energy under Contract No. DE-AC52-06NA25396 and by grants from the U.S. DOE under Contract No. DE-FG02-92ER40747 and the NSF under Contract No. NSF PHY-0852368.

\section{APPENDIX A: THE DATA MATRIX}

Data collected from $M$ monitors for $N$ measurements is organized in a data matrix of the form of Eq. (1). ICA and PCA model $\mathbf{x}$ as a linear combination of ICs and PCs, respectively. ICA and PCA of $\mathbf{x}$ result, respectively, in modes and patterns, which are associated with the source signal's strength in space and time.

\section{APPENDIX B: INTRODUCTION TO PCA}

PCA identifies patterns in data and expresses the highdimensional data by highlighting the underlying structures represented as PCs. The PCs are used to compress data by reducing redundant dimensions without much loss of information. PCA minimizes the redundancy measured by covariance, maximizes the signal measured by variance, and results in uncorrelated PCs. Two random variable vectors $\vec{y}_{1}$ and $\vec{y}_{2}$ are uncorrelated if their covariance is zero,

$$
\operatorname{cov}\left(\vec{y}_{1}, \vec{y}_{2}\right)=\left\langle\vec{y}_{1}, \vec{y}_{2}\right\rangle-\left\langle\vec{y}_{1}\right\rangle\left\langle\vec{y}_{2}\right\rangle=0,
$$

where $\langle\cdots\rangle$ is the expectation value. The core of PCA is singular value decomposition (SVD). SVD of the data matrix $\mathbf{x}(M \times N)$ yields eigenvectors $\mathbf{U}(M \times M)$ in column space and $\mathbf{V}(N \times N)$ in row space connected by a diagonal matrix of SVs $\boldsymbol{\Lambda}(M \times N)$,

$$
\mathbf{x}=\mathbf{U} \boldsymbol{\Lambda} \mathbf{V}^{T} .
$$

The columns of $\mathbf{U}$ span column space, the $M$-dimensional space of monitor number, and are called spatial patterns. The columns of $\mathbf{V}$ span row space, the $\mathrm{N}$-dimensional space of measurement number, and are called temporal patterns. The PCs are ordered by their $\mathrm{SVs}$, which represent their strength.

\section{APPENDIX C: INTRODUCTION TO ICA}

The objective of ICA is to calculate the $L$ source signals $\mathbf{s}(L \times N)$ given the data matrix $\mathbf{x}(M \times N)$, but the mixing matrix $\mathbf{A}(M \times L)$ is unknown,

$$
\mathbf{x}=\mathbf{A s} .
$$

ICA assumes independent source signals, a stricter requirement than PCA. Two random variable vectors $\vec{y}_{1}$ and $\vec{y}_{2}$ are independent if the covariance of any function of $\vec{y}_{1}$ and any function of $\vec{y}_{2}$ is zero,

$$
\left\langle f\left(\vec{y}_{1}\right), g\left(\vec{y}_{2}\right)\right\rangle-\left\langle f\left(\vec{y}_{1}\right)\right\rangle\left\langle g\left(\vec{y}_{2}\right)\right\rangle=0 .
$$

For time series data, source signal independence is related to diagonality of covariance matrices [11]. The autocovariance of a signal is $\operatorname{cov}\left[\vec{y}_{i}(t), \vec{y}_{i}(t-\tau)\right]$, where $\tau$ is a time lag, $\tau=0,1,2, \ldots$. Similarly, the covariance between two signals is $\operatorname{cov}\left[\vec{y}_{i}(t), \vec{y}_{j}(t-\tau)\right]$, where $i \neq j$. Applying these two results to mean-zero signals for reasons to become evident later, we write the time-lagged covariance matrix

$$
\mathbf{C}_{y}(\tau)=\left\langle\mathbf{y}(t) \mathbf{y}(t-\tau)^{T}\right\rangle .
$$

Source signal independence requires the time-lagged covariance matrices $\mathbf{C}_{s}(\tau)=\left\langle\mathbf{s}(t) \mathbf{s}(t-\tau)^{T}\right\rangle$ be diagonal,

$$
\left\langle\mathbf{s}_{i}(t) \mathbf{s}_{j}(t-\tau)^{T}\right\rangle=0, \quad i \neq j, \quad \tau=0,1,2, \ldots
$$

It follows that $\mathbf{A}^{-1} \mathbf{x}$ must also possess diagonal timelagged covariance matrices. The BSS problem is solved by obtaining a demixing matrix that diagonalizes the timelagged covariance matrices of $\mathbf{x}$.

The zero time-lagged covariance matrix $\mathbf{C}_{x}(\tau=0)$ does not contain enough information to obtain the mixing matrix A. The key is to utilize the additional information contained in the time-lagged covariance matrices $\mathbf{C}_{x}(\tau)$. Including more than one time lag improves ICA's performance by resolving degenerate SVs, but it introduces an additional complication of simultaneously diagonalizing many $\mathbf{C}_{x}(\tau)$. A technique for simultaneously diagonalizing several matrices with Jacobi angles is discussed in Ref. [16]. Typically, 20-50 time lags are required to separate source signals with close SVs $[4,5]$. We use the ICA algorithm second order blind identification (SOBI) [11], which accommodates multiple time lags, in our analysis and outline the algorithm below.

\section{Whitening}

The data matrix $\mathbf{x}$ is preprocessed to obtain mean-zero, whitened $\left(\mathbf{y y}^{T}=\mathbf{I}\right)$ data. Mean-zero data, which simplifies the covariance matrix calculation, is calculated by subtracting the average over the temporal variation. SVD is applied to the zero time-lagged covariance matrix of the mean-zero data matrix $\overline{\mathbf{x}}$,

$\mathbf{C}_{\bar{x}}(0)=\left\langle\overline{\mathbf{x}}(t) \overline{\mathbf{x}}(t)^{T}\right\rangle=\left(\mathbf{U}_{1}, \mathbf{U}_{2}\right)\left(\begin{array}{cc}\boldsymbol{\Lambda}_{1} & 0 \\ 0 & \boldsymbol{\Lambda}_{2}\end{array}\right)\left(\begin{array}{c}\mathbf{U}_{1}^{T} \\ \mathbf{U}_{2}^{T}\end{array}\right)$,

where $\Lambda_{1}$ and $\boldsymbol{\Lambda}_{2}$ are diagonal matrices of SVs separated by a cutoff threshold $\lambda_{c}$ such that $\min \left(\operatorname{diag}\left[\Lambda_{1}\right]\right) \geq \lambda_{c} \geq$ $\max \left(\operatorname{diag}\left[\boldsymbol{\Lambda}_{2}\right]\right)$. The cutoff threshold $\lambda_{c}$ is determined by the number of SVs $L$ included in the analysis. $\mathbf{U}_{1}$ and $\mathbf{U}_{2}$ are eigenvectors corresponding to $\boldsymbol{\Lambda}_{1}$ and $\boldsymbol{\Lambda}_{2}$, respectively. The mean-zero, whitened data is calculated

$$
\mathbf{z}=\mathbf{Y} \overline{\mathbf{x}},
$$

where $\left\langle\mathbf{z z}^{T}\right\rangle=I, \mathbf{Y}=\boldsymbol{\Lambda}_{1}^{-1 / 2} \mathbf{U}_{1}^{T}$, and $\boldsymbol{\Lambda}_{1}^{-1 / 2}$ indicates the inverse square root of the diagonal elements individually. 


\section{Joint diagonalization}

The time-lagged covariance matrices of the mean-zero, whitened data matrix $\mathbf{z}$ are calculated for a set of time lags $\left(\tau_{k}, k=0,1, \ldots, K\right)$

$$
\mathbf{C}_{z}\left(\tau_{k}\right)=\left\langle\mathbf{z}(t) \mathbf{z}\left(t-\tau_{k}\right)^{T}\right\rangle .
$$

Modified time-lagged covariance matrices $\overline{\mathbf{C}}_{z}\left(\tau_{k}\right)$ are constructed from $\mathbf{C}_{z}\left(\tau_{k}\right)$,

$$
\overline{\mathbf{C}}_{z}\left(\tau_{k}\right)=\left[\mathbf{C}_{z}\left(\tau_{k}\right)+\mathbf{C}_{z}\left(\tau_{k}\right)^{T}\right] / 2 .
$$

SVD is well defined, since $\overline{\mathbf{C}}_{z}\left(\tau_{k}\right)$ is real and symmetric,

$$
\overline{\mathbf{C}}_{z}\left(\tau_{k}\right)=\mathbf{W D}_{k} \mathbf{W}^{T} \text {, }
$$

where $\mathbf{W}$ is the unitary demixing matrix and $\mathbf{D}_{k}$ is a diagonal matrix. The Jacobi angle technique discussed in Ref. [16] is used to find the demixing matrix $\mathbf{W}$, which is a joint diagonalizer for all $\overline{\mathbf{C}}_{z}\left(\tau_{k}\right)$. The mixing matrix $\mathbf{A}$ and the source signals $\mathbf{s}$ are calculated

$$
\mathbf{A}=\mathbf{Y}^{-1} \mathbf{W} \text { and } \mathbf{s}=\mathbf{W}^{T} \mathbf{Y} \overline{\mathbf{x}} .
$$

The columns of A span column space, the $M$-dimensional space of monitor number, and are called spatial modes. The rows of $\mathbf{s}$ span row space, the $N$-dimensional space of measurement number, and are called temporal modes. The $i$ th IC represents the meanzero signal constructed by multiplying the $i$ th column of $\mathbf{A}$ and the $i$ th row of $\mathbf{s}$,

$$
\mathbf{z}_{\mathrm{IC}_{i}}=\vec{A}_{i} \cdot \vec{s}_{i}
$$

[1] A. Hyvärinen, J. Karhunen, and E. Oja, Independent Component Analysis (John Wiley \& Sons, New York, 2001).

[2] J. Irwin, C. X. Wang, Y. T. Yan, K. L. F. Bane, Y. Cai, F.-J. Decker, M. G. Minty, G. V. Stupakov, and F. Zimmermann,
Phys. Rev. Lett. 82, 1684 (1999); C. X. Wang, Ph.D. thesis, Stanford University, 1999.

[3] X. Huang, S. Y. Lee, E. Prebys, and R. Tomlin, Phys. Rev. ST Accel. Beams 8, 064001 (2005); X. Huang, Ph.D. thesis, Indiana University, 2005.

[4] F. Wang and S. Y. Lee, Phys. Rev. ST Accel. Beams 11, 050701 (2008); F. Wang, Ph.D. thesis, Indiana University, 2008.

[5] X. Pang and S. Y. Lee, J. Appl. Phys. 106, 074902 (2009); X. Pang, Ph.D. thesis, Indiana University, 2009.

[6] J. Kolski, Ph.D. thesis, Indiana University, 2010.

[7] J. Kolski, R. Macek, R. McCrady, and X. Pang, in Proceedings of the IPAC2012, New Orleans, Louisiana (IEEE, New York, 2012), WEPPR038 [http://accelconf .web.cern.ch/accelconf/IPAC2012/papers/weppr038.pdf].

[8] J. Kolski, R. Macek, R. McCrady, and X. Pang, in Proceedings of the ICAP2012, Rostock-Warnemunde, Germany, FRABI1 [http://accelconf.web.cern.ch/ accelconf/ICAP2012/papers/frabi1.pdf].

[9] C.X. Wang, Phys. Rev. ST Accel. Beams 7, 114001 (2004).

[10] A. V. Petrenko, A. A. Valishev, and V. A. Lebedev, Phys. Rev. ST Accel. Beams 14, 092801 (2011).

[11] A. Belouchrani, K. Abed-Merain, J.F. Cardoso, and E. Moulines, IEEE Trans. Signal Process. 45434 (1997).

[12] L. S. Walling, D. E. McMurry, H. A. Thiessen, and D. Neuffer, in Proceedings of the 1989 Particle Accelerator Conference, Chicago, IL (IEEE, New York, 1989), Vol. 3, pp. 1568-1570.

[13] H. Kwak, LeCroy Corporation (private communication).

[14] C. Beltran, A.A. Browman, and R. J. Macek, in Proceedings of the 20th Particle Accelerator Conference, Portland, OR, 2003 (IEEE, New York, 2003), TOPD004 [http://accelconf.web.cern.ch/ accelconf/p03/PAPERS/TOPD004.PDF]; C. Beltran, Ph.D. thesis, Indiana University, 2003.

[15] R. McCrady, R. Macek, and M. Prokop, Report No. LANSCE-ABS 06-008, PSR 06-002.

[16] J.F. Cardoso and A. Souloumiac, SIAM J. Matrix Anal. Appl. 17, 161 (1996). 